\title{
Design and Evaluation of a Soft and Wearable Robotic Glove for Hand Rehabilitation
}

\author{
Stuart Biggar \\ Biomedical Engineering \\ University of Strathclyde \\ Glasgow, United Kingdom
}

\author{
Dr Wei Yao \\ Biomedical Engineering \\ University of Strathclyde \\ Glasgow, United Kingdom
}

\begin{abstract}
In the modern world due to an increased aging population hand disability is becoming increasingly common. The prevalence of conditions such as stroke is placing an ever growing burden on the limited fiscal resources of health care providers and the capacity of their physical therapy staff. As a solution, this paper presents a novel design for a wearable and adaptive glove for patients so that they can practice rehabilitative activities at home, reducing the workload for therapists and increasing the patient's independence. As an initial evaluation of the design's feasibility the prototype was subjected to motion analysis to compare its performance with the hand in an assessment of grasping patterns of a selection of blocks and spheres. The outcomes of this paper suggest that the theory of design has validity and may lead to a system that could be successful in the treatment of stroke patients to guide them through finger flexion and extension, which could enable them to gain more control and confidence in interacting with the world around them.
\end{abstract}

Keywords-Hand rehabilitation; adaptive; variable; soft robotics.

\section{INTRODUCTION}

In the modern world an extended life expectancy coupled with a sedentary lifestyle raises concerns over long term health in the population. This is highlighted by the increasing incidence of disability stemming from multiple sources, for example medical conditions such as cancer or stroke [1]. Whilst avoiding the lifestyle factors that have a high association with these diseases would be the preferred solutions of health services the world over, as populations gets progressively older and more sedentary, this becomes increasingly more difficult [1], [2]. The treatment of these conditions is often complex, in stroke for example, the initial incident is a constriction of blood flow in the brain which in turn damages the nervous system's ability to communicate with the rest of the body. This damage will occur in one hemisphere of the body, but can impact both the upper and lower limbs, as well as impairing functional processes such as speech and cognitive thinking.

Physical therapy has been shown to help in repairing some of this damage, with additional benefits the sooner that this therapy can begin [3], with studies suggesting that accurate projections of stroke recovery can be made as soon as 4 weeks post stroke [4]. The therapy process has the drawback of being very intensive for the therapist to conduct coupled with the fact that they can only attend directly to one patient at a time, the intensity of this helper role places considerable strain on them mentally as well as physically [5], [6], [7]. When viewed alongside the increasing numbers of patients it is inevitable that this necessity will start to apply considerable strain on healthcare budgets in the coming future. A mechanical solution should then in theory enable therapists to engage with this increasing number of patients without the physical burden of additional therapy. Therapists would then be able to perform a more prescriptive role whilst the device takes care of the manual tasks. Traditionally attempts at therapy are focussed on the legs and getting the patient independently mobile, however this does not enable the patient to become fully independent, as the hand receives limited focussed attention. This perception was supported by our own consultation with stroke patients in various locations around central Scotland. It has been shown that is important to make sure that every muscle is treated to avoid a directionally dependent recovery [8].

These factors suggest that it may be possible to improve the outcomes of hand physical therapy by providing patients with an artificial hand aid to assist them by guiding their movement and performing the role of exercising the hand during the early stages of recovery. There are a variety of designs that have been developed in this field. More traditional designs use a mounted system, [9], [10], [11], [12], for these the patient would be required to sit down with little scope for movement, this may benefit them in focussing on the task in their therapy, but also has the drawback of being restrictive as an advanced or more confident patient would be unable to practice moving around and using their hand in tandem.

These strengths and weaknesses are repeated in systems that are not secured in place, but are too large and bulky to carry around [13], [14]. These systems are also too large and costly to be set up in patient's homes, as a result they kept to specialist facilities [15], [16]. Whilst this enables multiple patients access to the device, it also means that an individual patient only has access to it at set times, scheduling for which may present a challenge to incorporate the individual factors of their life, such as travelling time, fatigue or personal 
factors. This can also be restrictive for patients who live in rural communities, where access to the resources will not be as readily available.

Exoskeletons are not the only method that can be utilised, with theories such as Virtual Reality being used to show improvements in patient mobility [17], [18]. However if the intention is to provide a patient with a system that they can utilise at home, these is perhaps impractical as it raises questions of operating cost, space and timescales, as these programs require a rigorous training program of 5 hours per day for 9 nine days.

From this the objective was to develop a design and prototype of an exoskeletal hand that is both lightweight and functional, enabling disabled patients the opportunity to choose to exercise their fingers in a manner that better suits them, such as whilst also walking around or when in a seated position. If this flexibility were possible then stroke patients for example may be able to conduct a portion of their assisted therapy at home, which may improve engagement and potentially their outcomes as well.

Robotic exoskeletons are the modern approach to supporting physical therapy firstly because they provide a rigid shape to improve patient posture, along with a solid frame for which to place the components that will be used to guide and drive movements. There are two traditional approaches for their design, placing their emphasis on either functionality or aesthetics, although now modern demands try to integrate as much of both systems as possible [19]. Our discussions with patient groups found that they were very eager for any system that would enable them to practice movement independently, preferably in their own home.

There are even models with computer control available, with provisional testing showing a good repeatability of activity [20]. Whilst the glove is of a size that it could enable a patient to wear it whilst performing other tasks the supporting computers required to operate it make this device impractical for everyday use. It will also require a staff member to be on hand to adjust the components to fit the wearer. Devices can be programmed in advance by the therapist to repeat a pattern of movements [21]. The preliminary data shows a strong repeatability from the system in comparison to the therapist motions. The main risk for a system of this nature to be used in a domestic setting is in extreme responses in the patient base, where some may be demotivated by the lack of control the system gives them, whilst may be overly enthusiastic and risk harming themselves, this is a particularly big risk when using an automated system with no therapist to supervise.

Another popular method of control for an exoskeleton is with electromyography (EMG), where the electrical impulses of the muscle can be used to directly control movement [22], [23]. In a condition such as stroke, where there is damage in the connection between the brain and the body, achieving this for one patient can be very challenging and would require individual programming for each patient with no guaranteed level of output. EMG has further issues for control of the hands as the muscles used to control the fingers are slender and overlap with the arms, this can make differentiating clear signals very difficult, particularly if the muscles are damaged. Attempts have been made to work around this problem with ideas such as signal mirroring, where the functional arm would be used to make commands [24]. This theory unfortunately limits the activities that the hands can perform.

Full arms as well as full body systems have been devised previously, such as the HAL-5 [25], these enable the patient to exercise more than one joint at a time, however they lack specialist components to give the user full control of grasping their hand. Many full arm systems will only provide the operator with a bar to hold onto whilst performing the arm motions, this does not provide an opportunity for the patient to also practice grasping as a repeated measure [26], [27].

Mechanical systems have the common factor of being too heavy to be practical to offer for a patient to use for home therapy. Consequently if the intention is to look for a device that a patient could utilise at home, it needs to be both lightweight and easy to put on. These needs favour a cable driven set up, which has been trialled previously [28], this set up has flaws in terms of protecting the operating mechanism, with many components exposed to the possibility of impact in use. This means that if the device were provided for patients at home they may incur further costs and inconvenience having to get it repaired.

Current hand exoskeletons require additional bulk to make them adjustable for the varied structures of the hand, as the length of bones and thickness of muscles vary from person to person. Using a single standard size would result in the joint centres not being accurately placed and subsequently inefficient when used on the whole stroke population. The consequence of this is that the device would then be too heavy and cumbersome for the patient to wear whilst walking around.

In this paper we will introduce a new design to address the issues highlighted by previous designs. The design was developed with the key aims of providing good repeatability of movement in a system that is both lightweight and low cost. In the next section the design and developed prototype will be outlined, this will be followed by a description of our assessment process and an analysis of the outcomes.

\section{DESIGN AND PROTOTYPE DEVELOPMENT OF THE GLOVE}

\section{A. Design of the glove}

This concept was influenced by looking at nature, thinking about how an octopus is capable of wrapping around objects and moving in sync with them. It was considered that this principle of suction based gripping could be applied to make a glove that could tightly fit the patient's hand without the need for a bulky metal frame.

To address the issue of weight it was considered that it could be possible to develop a system that would utilise the patient's own skeleton as the frame to operate from, this would limit the need for large, bulky components that would need to be secured to the patient. The concept for this design was that a glove would provide a tight fitting base that matches the shape of the hand. However a glove is not able to 
power motion so would need an additional actuator to be fitted, in turn making it difficult for a glove that can be donned and doffed by the user to have a stable base for the actuator to function consistently. This would result in the same action producing different outputs from the mechanism due to variation in its position, and the solution to this is to position the actuators externally on a more level surface. The actuator can then control the motion with a cable driven system that would act in the same manner as the conventional muscle tendons of the hand (Fig. 1). This set up would make the device easier to don and doff as well as distributing the weight, making the hand component lighter and therefore less likely to weight down the arm. The glove would be held in place during action by suction cups, where a dual layered glove could have a vacuum created to tightly fit the shape of the hand. The vacuum also enables one size of glove to securely fit hands of a range of sizes rather than needing to be adjusted for each user.

Multiple options were reviewed on what was the best way to create the vacuum to ensure the strongest grip. The devised solution was that there would be a two layered glove as shown in Fig. 2, with the air gap in between being sucked out to create the vacuum. This however only serves to make a glove with double the thickness, so the inner glove would have holes cut into it which the suction cups would then be threaded through. When the air is extracted these cups will then fix the glove to the skin before the actuators move the joints. If the suction cups were fixed in place against the skin it would provide the firmest base for the vacuum generation to be performed, however once the vacuum is released, it would not allow the skin to expand back to its natural shape after the contraction created by the vacuum, subsequently damaging it.

The driving mechanism is required to be between the layers firstly to protect the mechanism from damage that may occur in making contact with objects they are interacting with and secondly to lessen the deformation on the outer layer of the fingers to maximise the surface area for gripping. The most practical mechanism to control motion would be a cable driven system with an external motor actuator. This reduces the weight in two ways, firstly as the components are lighter,

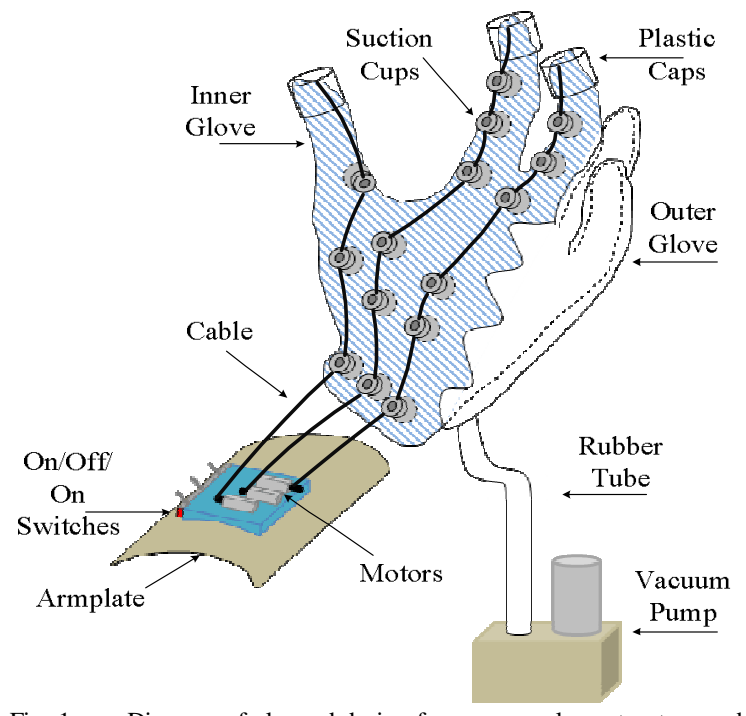

Fig. 1. Diagram of planned design for vacuum glove structure and required components. and secondly that the mechanism for driving the cable motion can be moved away from the hand, distributing the remaining weight.

This creates an issue where the downward pressure from the outer layer of the glove will press down and constrict the movement of the driving mechanism, or alternatively the mechanism may damage the material of the glove. The solution to this was to thread the cable mechanism through a series of segments of plastic tubing as well as the suction cups, preventing downward pressure constricting the cable and removing the friction between the cable and gloves. It also allows a straight path to be formed between the end of the finger and the motor, minimising the horizontal forces on the cable for more efficient performance. The suction cups also serve the secondary purpose of acting as a guide for the cable, as segments of plastic tubing (approximately $1 \mathrm{~cm}$ in length) were positioned through the suction cups to direct the cables between the motor and the anchor point in the fingertip. The fingertips were made from plastic caps from small drinks bottles, as they were a similar size to the distal phalange of the fingers, providing a fit that was not excessively tight for the wearer whilst also allowing them to be able to feel contact.

For the fingers the cups were positioned on the palmar side of the first two phalanges of the fingers, whilst the end of the cable was anchored to the plastic cap, meaning that when the motor contracts the cable the threaded between the cups shortens. With both ends anchored in place, as the cable shortens it will firstly apply a force pulling the cup away from the finger in an attempt to form the most direct route possible between all of the cups and the anchor point. This force is cancelled out by a combination of the vacuum force pulling it towards the finger and the force that the material applies to hold the cup in position. This results in rotation of the finger joints as the only solution to reducing the length of cable without changing the position of the cups (Fig. 2), replicating finger flexion.

The cups were positioned to the centre of the phalanges of the fingers along with the areas of the body of the hand that do not bend when flexing the fingers, this was to ensure that motion firstly did not cause the vacuum to break as well as to prevent the cups from impeding the movement of the joints. The motion of the finger joints was limited to a single motion action for three fingers (thumb, index and middle) that are required for grasping. The final two fingers of the hand were not motorised as they predominantly perform a supporting role rather than a grasping one and the device is intended to perform the therapy role of flexing the fingers. This motorised design helps the user to perform both a two-finger and threefinger pinch grip that can enable patients to interact with many

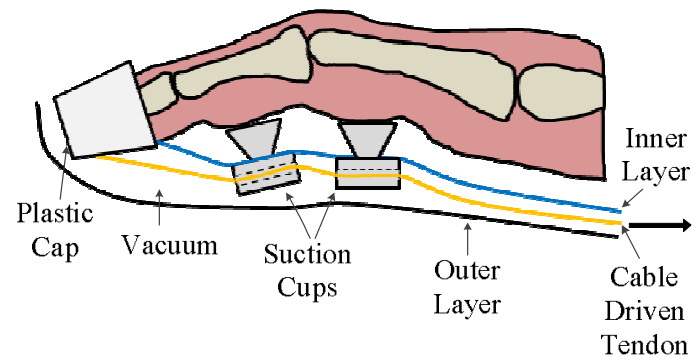

Fig. 2. Demonstration of the vacuum principle that will be used to move the finger. 
everyday objects.

The motion was planned to be driven by a set of $12 \mathrm{~mm}$ micro motors, with one for each finger. These motors were then to be secured to an arm plate that could wrap around the wearer's forearm; this unit would also have the control switches attached to it (illustrated in Fig. 1 and photographed in Fig. 3b). The motors had a rated torque of $60 \mathrm{mNm}$, a speed of $51 \mathrm{rpm}$ and an output power of $588 \mathrm{~mW}$. This will be sufficient to overcome the level of static passive joint torque projected by Kamper [29]. The power supply for these parts would be stored away from this plate to keep it lightweight, as a small belt attachment. The same principle was intended for the location of the pump, keeping the heaviest components of the design away from the arm to stop it dragging the user's arm downwards.

Finger extension was powered by an elastic band that was stitched onto the back of the hand, as the fingers flex the elastic band would stretch and store potential energy that would be released once the motor's reverse setting is selected to slacken the cable, this enabled the fingers to quickly extend back to their initial position.

\section{B. Prototype Development}

With an established concept of the design, the next stage was to develop a working prototype. The vacuum layers were formed from rubber gloves, cut to an appropriate length with vertical incisions into the smaller inner glove for the suction cups to be positioned. They were placed on the palmar side of the hand, away from areas of skin that contracts during flexion. The cups themselves were constructed of a siliconerubber compound and were then secured in place with rubber cement. Further incisions were then made on the cups to allow the plastic tubing to be threaded through to support the cable, no adhesives were necessary to secure the tubing in place as the cup itself provided enough to constrict movement of the tubing.

The tubing connections between the cups were stitched into the inner layer to ensure they would not move under loading, as were the elastic bands. They were secured to each finger segment to ensure that the full finger extended when the tension was slackened in the cable.

The motor housing was a custom-designed 3D printed unit that was screwed onto an arm plate, providing a base for the motors and their operating switches to be secured in place. Each motor was operated by an on-off-on switch to give the wearer the capacity to control flexion and extension. They were wired so that if the switches were pointed proximally towards the user the cables would contract to flex the fingers and extend when directed distally towards the hand. To reduce the total weight of the system the design was limited to three motors to enable movement of the thumb and first two fingers as these fingers are predominantly used in grasping activities, with the remaining fingers often supporting a raised object. Each motor was fitted with a miniature pulley for the cable to coil around.

The arm plate that was used was a shin guard for contact sports (youth size) as it was a good fit for the length of the

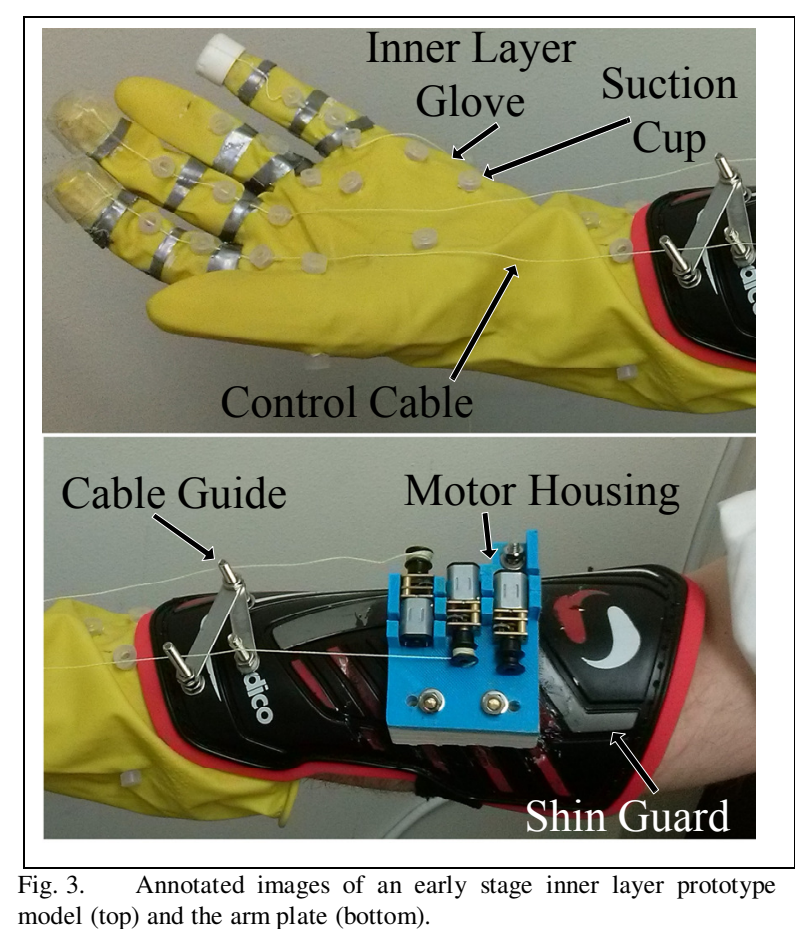

forearm and came fitted with a Velcro strap to secure it in place. The motor housing unit was custom designed and 3D printed before being screwed into this shin guard with additional screws being fitted vertically to one end of the shin guard to act as cable guides to prevent a crossover of the cables and subsequent tangling.

The vacuum was powered by a small air pump secured to the glove by rubber tubing. This tubing was in turn glued by rubber cement into position between the inner and outer gloves. The vacuum pump was separated from the rest of the device to reduce the total worn weight that would be applied to the wearer's arm. Once assembled the inner and outer layers were sealed together at the base by rubber cement.

Once the user had donned the device to use it a Velcro strap was wrapped around their wrist to ensure that no air could get in to disrupt the vacuum. Images of the internal mechanics and shin guard set-up are shown in Fig. 3 with the final model in Fig. 4 and a demonstration of being worn in Fig. 5.

\section{EVALUATION}

\section{A. Feasibility Study}

To assess the effectiveness of the developed design it is important to try and recreate the patterns of movement that a person without a disability would use when interacting with objects of differing shapes and sizes. This was achieved by performing motion analysis of the fingers both with and without the device when it was used to grasp and lift a selection of small to medium sized spheres and cubes. These grasping tasks would be performed in a range of conditions to assess how the features of the glove impact the replicability of 


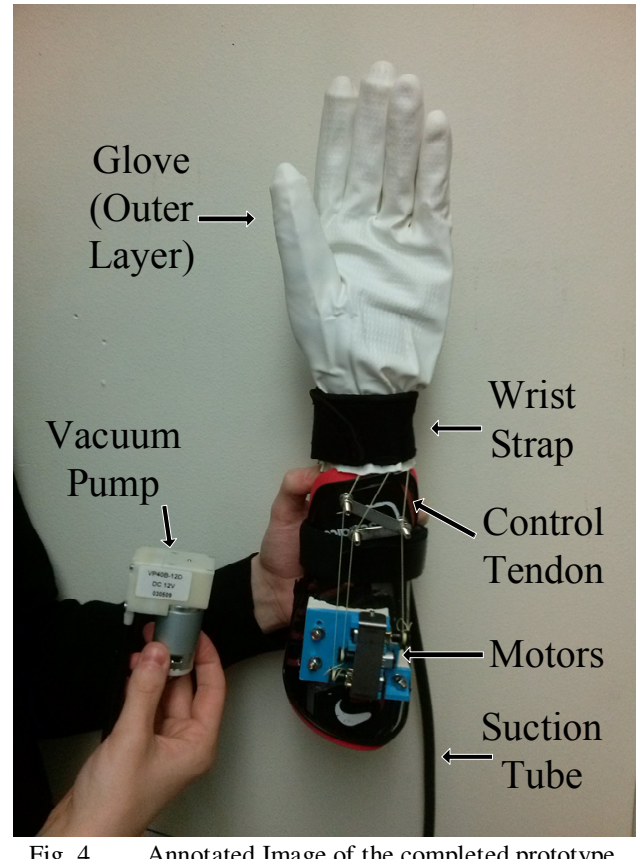

Fig. 4. Annotated Image of the completed prototype.

the hand's movement. These conditions were (1) using the hand without wearing the glove, (2) a baseline scenario where the hand would be used within the glove but there would be no motorisation of the joints to show if the structure of the glove alters how hand grasping is performed, (3) using the device whilst a healthy participant holds their hand limp and (4) using the device on a pose able 3D printed hand to simulate a user who had no control over their hand at all.

The items selected for grasping were drawn from the grasp and pinch test stages of the Action Research Arm Test (ARAT, [30]) as they were cubes and spheres suitable for lifting with one hand as the largest of these items is $10 \mathrm{~cm}$ in length. The final selection of items were; 1 large block $(10 \times 10 \times 10 \mathrm{~cm}), 1$ small block $(5 \times 5 \times 5 \mathrm{~cm}), 1$ cricket ball (rubber practice version) and 1 marble. These items were selected to test the primary function of the device (grasping), with a mix of weights and sizes.

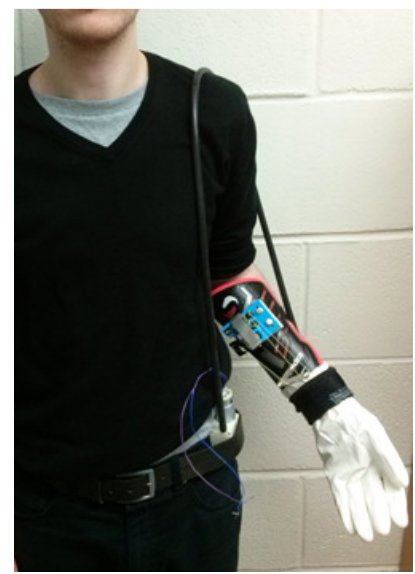

Fig. 5. Demonstration of how the device fits the user when worn.
For the testing the participant would take a seated position before placing their hand over the item, grasping it and raising it off the tables surface above their head and holding it in place for a count of two before returning it to the table and releasing it (each lifting task will be performed 3 times). The movements would be recorded by the Vicon motion analysis system with a tailored marker set used on the hands to monitor the angular changes in the finger joints (Fig. 6).

\section{B. Analysis}

The Vicon marker set used in this study compared the positional changes of the four markers that were positioned on the active fingers along with at the base of the hand, enabling it to calculate the angle at each joint during the recording period. It was a custom marker set collecting data from the first two fingers and thumb. This data was then filtered with a second order recursive digital filter. Visual inspection was performed of the recorded video data to assess which time period could be classed as the completed grasp.

\section{RESULTS}

Fig. 7 shows the changing relationship between the finger joints under each of the experimental conditions. The most notable outcome of the data is that when not wearing the prototype the MCP for the index finger does proportionally less work in lifting the large items (approximately 10-14\% of the fingers total rotation), with the exception of using the pinch grip for the marble, where the MCP makes the largest contribution to finger rotation $(37 \%)$. The difference in the action of the finger joints is shown fully in Fig. 8; this illustrates the increased rotation of the MCP to complete the grasping of the marble, whilst at the same time the DIP has a negligible change from its resting position.

This trend is repeated in the middle finger action of each joint across the activities, however the workload relationship changes for gripping the marble, where the index finger has a small inversion of its activity (with the largest contribution to movement coming from the MCP and the lowest at the DIP), however the middle finger has a very flat distribution of work between the finger joints (each joint performs between 32.3 and $34.9 \%$ of the total workload).

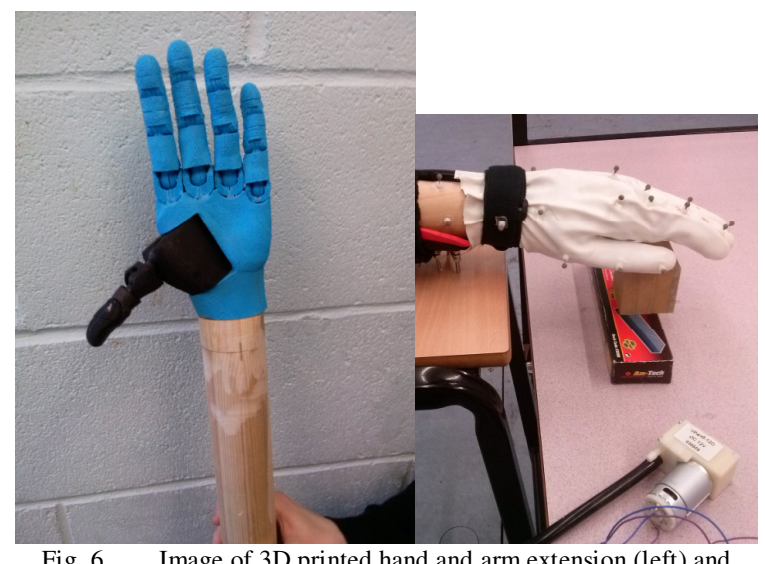

Fig. 6. Image of 3D printed hand and arm extension (left) and demonstration testing protocol (right). 


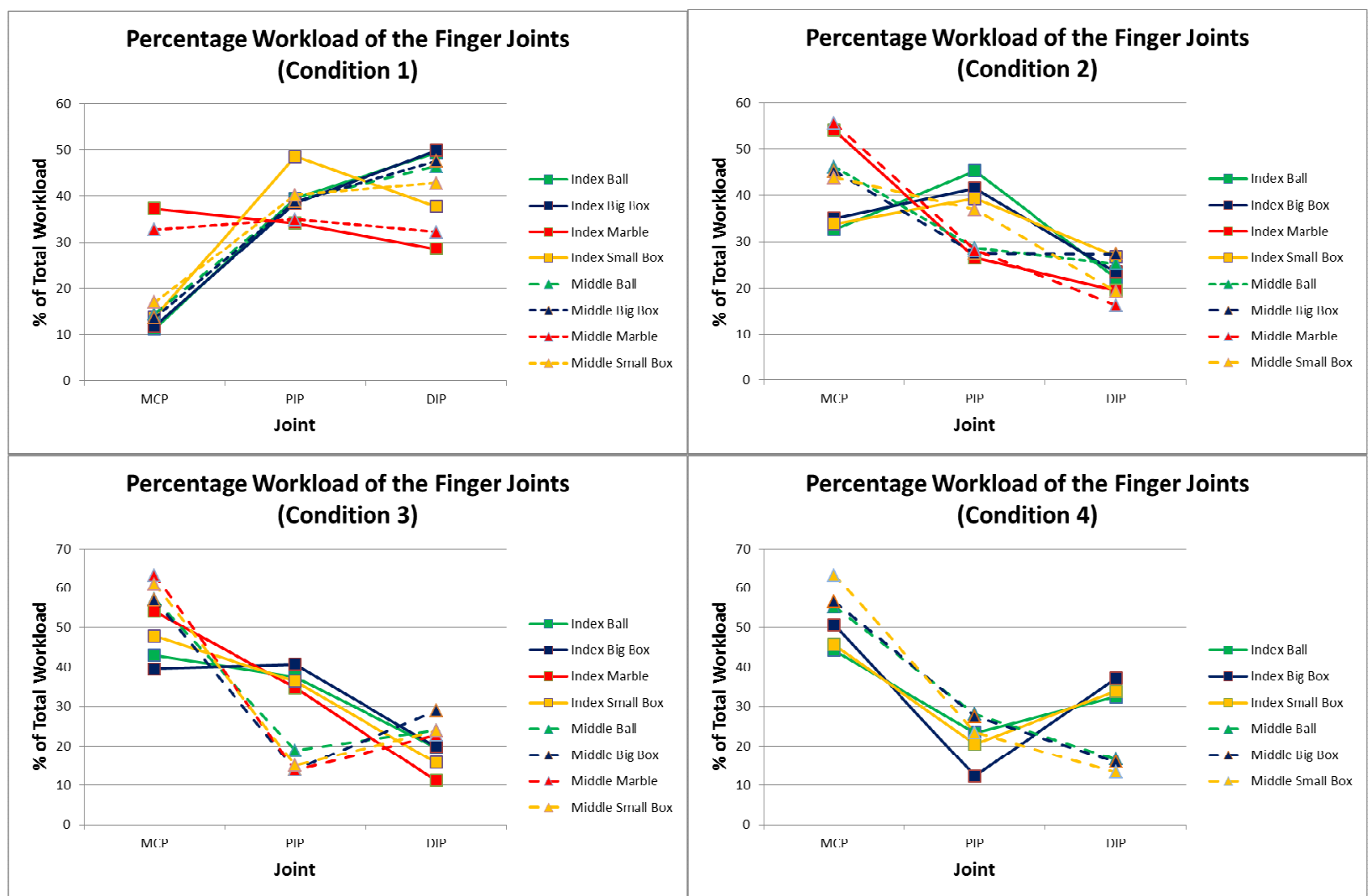

Fig. 7. Overview of the changes in percentage based workload distribution across the finger joints. Condition 1 (top left) shows the control condition of the user's hand when not wearing the device, condition 2 (top right) shows the performance when the user is wearing the device but not using the motors.

Condition 3 (bottom left) is when the user is operating the device as intended and condition 4 (bottom right) is the device being worn by the 3D printed hand. Square markers indicate the index finger and triangular markers indicate middle finger.

When the device is worn (as shown in Fig. 7 and 9-11) there is an inversion of the workload distribution by the finger, giving the fingers a profile closer to the pinch grip used to lift the marble, with an additional increase in activity of the PIP. The $3 \mathrm{D}$ printed hand was unable to grasp the marble in the device as the base of the thumb was positioned too distally on the $3 \mathrm{D}$ hand and consequently the thumb and two fingers were unable to form a 'pincer' shape to grasp the marble. The required position was achieved by a user wearing the device, suggesting that the error was due to the shape of the hand.

The findings show that there was a reduction of activity in the DIP when the glove was worn by a user, with a range of movement from $23.44-40.84^{\circ}$ for index finger without the device, dropping to $7.61-14.64^{\circ}$ with the device on. As the activity increases to $25.61-41.51^{\circ}$ for the $3 \mathrm{D}$ printed hand this suggests that the reduction may be due to the lack of cross joint coupling in the finger as each joint for the printed hand was independent. This principle of biomechanical coupling has been shown to be present in both modelling [31], [32] and can be factored into cable driven glove designs [33]. A cable driven mechanism would be expected to have an increased rotation at the distal end, as the DIP is the closest joint to the anchored end of the cable, which would contract first as the cable gets shorter under loading. Due to the changes in hand structure this places the rotational forces on the loosest fitting joint first. 


\section{DISCUSSION}

When testing without the prototype the marble is grasped with a different pattern of movement to the other objects. It is regarded as the pinch grip, where the fingers grasp an object without using the palm for support and is required due to a combination of the size and shape of the object being grasped. To maintain strength and consistency in this grip the finger joints rotate at similar angles, resulting in a straight line force being applied between the tips of the index and middle fingers with the thumb, giving the wielder better control of the marble. In the power grasp the metacarpals and proximal
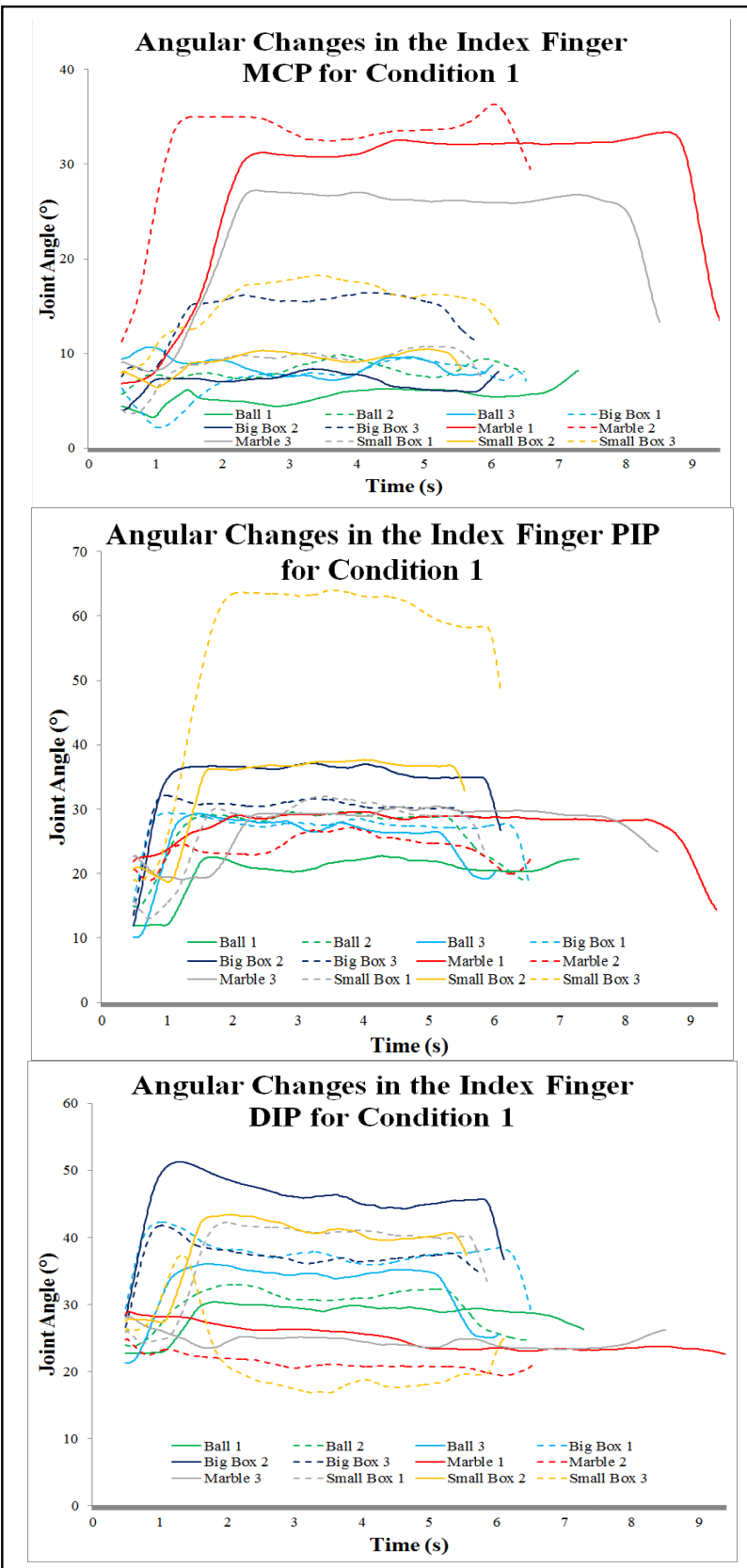

Fig. 8. Join $t$ angle variations in the index finger for grasping the four testing items without the device. The data is filtered for a moving average of 50 data points. phalange form a base whilst the distal phalanges wrap around the object and push into this base. This allows the fingers to create a greater force as control is not an issue when being pressed against the palm of the hand. With respect to the first testing condition, the differences between these patterns of movement are illustrated in Fig. 7 \& 8, where grasping of the marble is shown to require a higher degree of rotation in the $\mathrm{MCP}$, coupled with a reduction in rotation of the DIP. For the power grip activities the action of the middle finger replicates the pattern of movement by the index finger; however for the pinch the middle finger has a more even workload
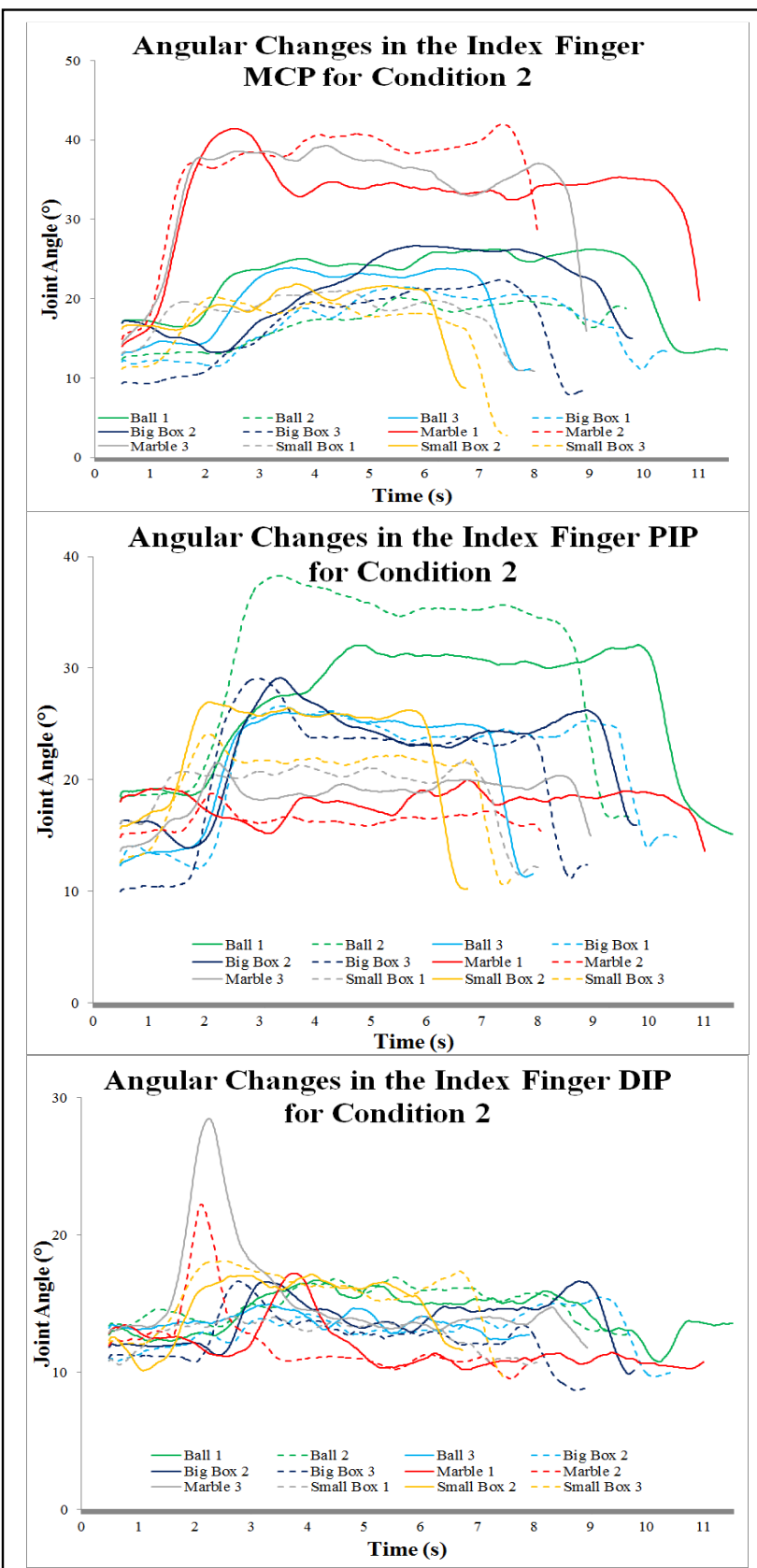

Fig. 9. Joint angle variations in the index finger for grasping the four testing items whilst wearing the device with no vacuum or motors used. The data is filtered for a moving average of 50 data points. 
distribution. This will be a result of the marble's small, spherical shape, where the majority of the grasping work will be performed between the thumb and index finger, leaving a supporting role for the middle finger to stop it from dropping.

When the device is worn by the user this pattern inverts for the index finger, where DIP activity drops and the MCP increases, for example when lifting the ball the DIP drops from $31.74 \pm 2.38^{\circ}$ in condition 1 to $15.08 \pm 1.2^{\circ}$ in the second, $14.64 \pm 2.24^{\circ}$ in the third and $21.48 \pm 2.92$ in the fourth, whilst the MCP increases from $7.22 \pm 1.61^{\circ}$ in the first condition to $22.24 \pm 3.17^{\circ}, 31.99 \pm 3.12^{\circ}$ and $29.21 \pm 1.6$ respectively (Fig. 811). This is counter-intuitive to the design, where the cabledriven system would be expected to have the greatest rotation at the DIP as the anchor that holds the cable in place is located there and would experience the brunt of the rotational forces. The increase in workload at the MCP is most likely a result of changes being made by the user in terms of their approach to the items due to the performance of the glove, where the unimpaired user may use a 3 fingered approach to gripping the ball, when using the printed hand the index and thumb grasped the central axis of the ball to prevent pushing it across the surface. Consequently the distal phalanges are pressed against the ball and do not rotate as much as they would otherwise, resulting in an increased activity of the MCP to complete the grasp. Ultimately the use of the glove is changing the approach the wearer would take to objects in their environment.

For conditions 1 and 2 there is no motorisation of the joints, it could therefore be assumed that there would be similar results in these conditions. Fig. 8 and 9 show that there is a reduction in both the PIP and DIP for the index and middle fingers with each object, this is likely contributed to by the double glove, as the inner layer will move with the finger through the rotation, but within the outer layer, giving the impression of reduced movement. That this anomaly in the performance repeats in the third condition (Fig. 10) but changes in the fourth would suggest that the glove was a poor fit for the user's hand, as the 3D printed hand used in condition 4 had a wider body and fingers, limiting the possibility for movement to occur that is not noticeable by the Vicon cameras and saw an increase in results for DIP (Fig. 11). The tip trajectory also decreases during this motion with an average speed of $74.13 \%$ rotation for the total grasping period when not wearing the device compared to $1.09 \%$ for the $3 \mathrm{D}$ printed hand. By contrast, when the user wore the device their average rotational speed was $3.66 \%$, suggesting that the larger printed hand provides a greater resistance within the device than the user keeping their hand limp. This places emphasis on ensuring that the outer glove is not too loose that the performance may become affected; this could be remedied by a tighter fitting glove or using a more flexible material in the outer layer.

The middle finger experiences an additional decrease in the peripheral interphalangeal joint, which may be due to the shape of the item, for example the cricket ball's spherical shape will provide a greater restriction to any attempt to rotate around its central point $\left(19.94 \pm 2.7^{\circ}\right.$ for the middle finger compared to $32.16 \pm 3.04^{\circ}$ for grasping the ball in the second condition as well as $14.87 \pm 5.39^{\circ}$ compared to $45.11 \pm 3.77^{\circ}$ for
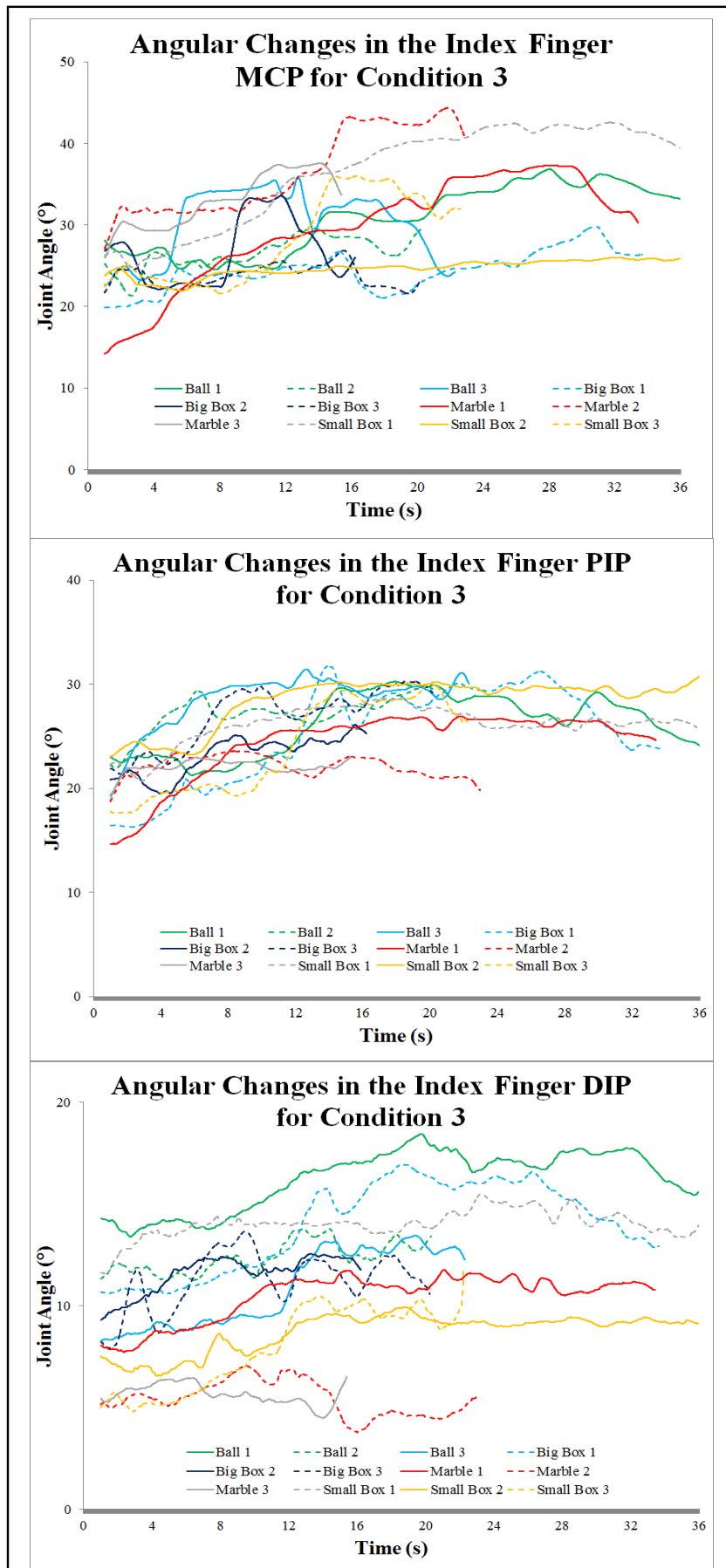

Fig. 10. Joint angle variations in the index finger for grasping the four testing items whilst wearing the device, keeping their hand limp. The data is filtered for a moving average of 100 data points.

the third and $16.34 \pm 2.31$ with $32.1 \pm 2.96$ for the fourth). When comparing the index and middle fingers, despite them being operated by identical components in the same mechanism, there is a clear discrepancy in their performance. This is likely to be due to the glove changing the technique of the wearer in grasping, for example the middle finger wrapping 

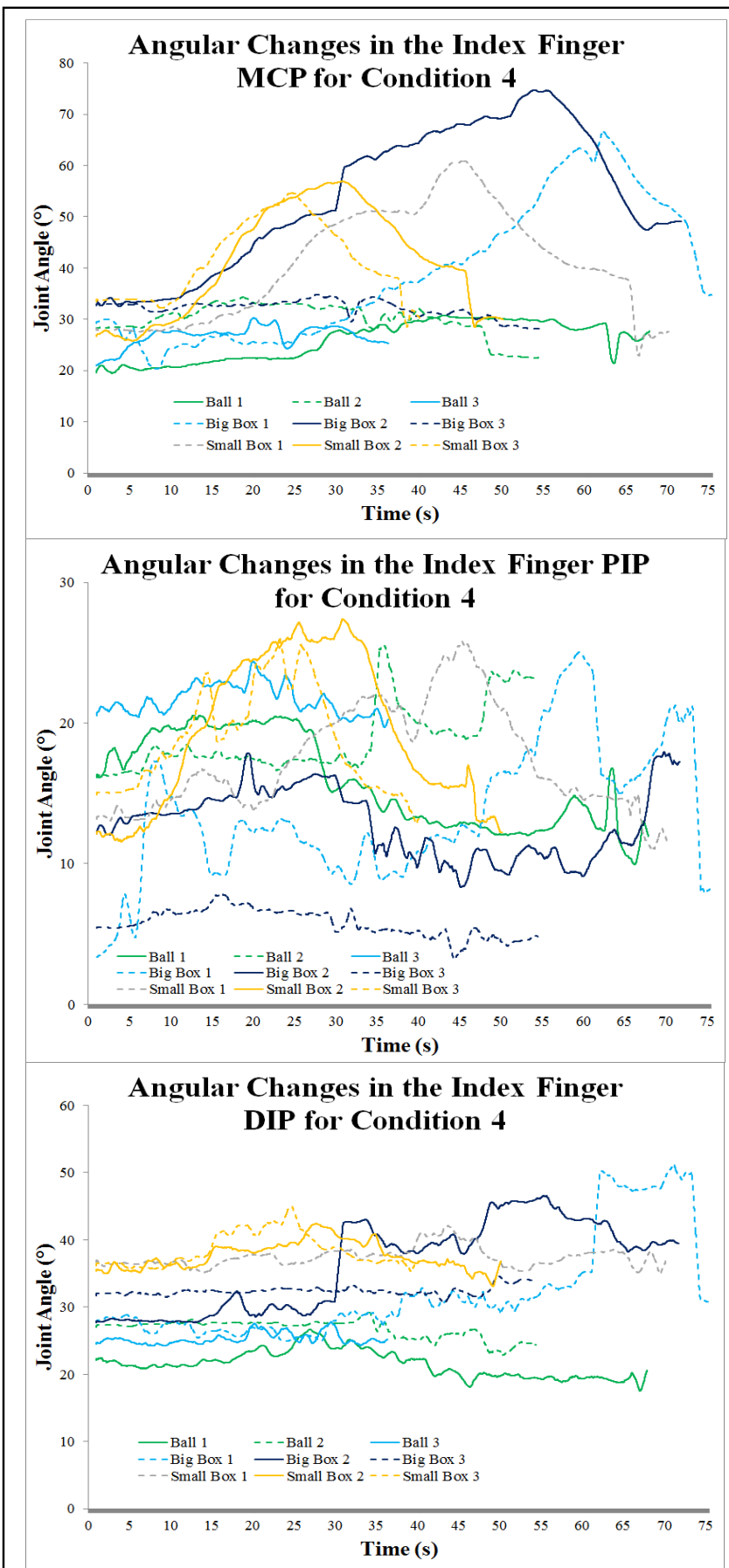

Fig. 11. Joint angle variations in the index finger for grasping the four testing with the $3 \mathrm{D}$ printed hand wearing the deviice. The data is filtered for a moving average of 100 data points.

around a wider part of the ball with the DIP and PIP coming into contact sooner, this obstruction stops the joint from rotating and results in an increase in the activity of the MCP to compensate for this. This will change the profile of the finger and may contribute to the irregular grasp pattern shown particularly by the middle finger in Fig. 7. When the 3D printed hand is used in Condition 4 this discrepancy between the fingers becomes more acute at the DIP, suggesting this is an issue of grasp technique prompted by the glove.

Technique was a critical factor for lifting the items, as the device was capable of grasping the $10 \mathrm{~cm}$ wooden block, provided it had been rotated to accommodate that the fingers were unable to stretch over the length of its sides, pinning the block from 3 directions, however when coupled with its weight the block became difficult to support for longer than a few seconds, causing it to fall not long after it cleared the table surface. At the opposite end, the marble's small size and smooth surface meant that the fingers were unable to effectively grasp it. A two finger pinch was possible on the marble, but due to its round surface and the rubber fingertips of the glove there was an insufficient friction force to support it. This was also an issue with grasping the rubber ball as the rubber-rubber surface contact was not stable, contributing to the adjustment made in where to grasp it when wearing the device. Achieving the correct grasping technique was more complicated with spherical objects as the first finger to make contact with the object would apply a force prompting it to roll out of position before the corresponding fingers could arrive to pincer it. This meant that correct grasping with the device also placed an emphasis on the timing of the finger movements.

The angular changes (as illustrated in Fig. 8-11) showed that there was a strong consistency within each condition, however there is a noticeable variation across the conditions, reinforcing the idea that the main catalyst for difference in the recorded data was due to the device changing the subject's interaction with the objects, as across the recorded conditions the sensations in the hand are different.

In terms of total rotation of the fingers there are significant differences noted between each condition except for conditions 2 and 3. When not wearing the glove the joints of the index finger complete $82.28 \pm 11.21^{\circ}$ of rotation compared to $68.6 \pm 9.62^{\circ}$ when only the glove is worn. When the user operates the glove as designed the total rotation of the fingers is $74.23 \pm 5.89^{\circ}$ and for the $3 \mathrm{D}$ printed hand this rose to $108.49 \pm 25.42^{\circ}$. The variation for the final condition would suggest that the 3D printed hand is used in different ways to grasp the various objects. As all of the testing conditions differ significantly from the control condition this supports the thought that the glove does adjust how the wearer grasps. Whilst the increased motion of the MCP may appear on first inspection to be increased to compensate for a reduction in the PIP and DIP, that this appears to be a false reading means that the $\mathrm{MCP}$ change in all of the recordings when wearing the glove must be due to a change in grasping technique, with the palm of the hand playing as larger role in supporting the lifted weight. This change in approach to the object will be an attempt to protect the cups in the fingers from taking the weight of the item and incurring strain damage.

The most obvious variation across the recordings is the change in the recording time, as the motors cannot function as quickly as the natural hand. Time was measured by visual inspection of the recording frame by frame to isolate the 
beginning and ending of grasping motion. The numbered frames were then matched to their corresponding times to assess the total time period. For the first testing condition all of the trials are completed within 10 seconds (with $75 \%$ being completed within 7), in comparison for the third condition $83 \%$ took over twenty seconds to complete with one taking over fifty seconds (this was due to poor grasping technique creating an error in positioning of the fingers, causing the small block to be unstable and be moved more slowly to avoid being dropped). To complete just the initial grasp took $0.68 \pm 0.4$ seconds in condition 1 , this increased to $8.47 \pm 4.5$ seconds in condition 3, or approximately twelve times as long. This shift in timescale is discouraging for using the system as a supportive device in daily activity, for example a patient is unlikely to want to approach a door, take over eight seconds to grasp the handle before interacting with the door and then taking another eight seconds to release it. The design will need to be revised before it could be used in supporting daily living, but could still be used in physical therapy. Possible improvements would need to be made to the performance of the motor, for example by increasing the torque.

Whilst an externally controlled mechanical system will never be able to bridge the time difference created by reaction time, the difference in rotational time would require a very high torque motor, however a compromise must be struck to ensure that the motors are not too large and bulky so that the user retains the capacity to move around whilst wearing the device. The prototype has been shown to be capable of lifting small and medium sized objects and it is believed that with small revisions to the design could be used to help patients who have experienced a hand disability to perform rehabilitative exercises and in the future regain independence.

\section{CONCLUSION}

In response to the increasing strain of physical disability on the medical field there are possible mechanical solutions to ease the therapist's workload. A principle theory for a cable powered vacuum glove was devised which was then developed into a functional prototype. To test the device the experimental protocol was developed to assess if this would replicate motion or alter it. Our findings suggest that the shape and structure of the glove does alter the recorded grasp pattern, a change that is likely to be result of a combination of the changed grasp position of the hand due to the suction cups being pressed against it as well as the stiffness of the outer material effecting the measurement. However whilst it is a change from the instinctual power grasping pattern, it bears similarity to an exaggerated version of the pinch grasp, with a larger percentage contribution from the MCP (rising from 11.21 to $42.9 \%$ for grasping a cricket ball) and a reduction in contribution from the DIP (falling from 49.28 to 19.64\%). Exaggerated motion can be beneficial in physical therapy to recover independent motion. There is also a twelve-fold increase in the time to complete a grasp. Ultimately the design encouraged the wearer to use more of a clamping action in grasping. It is an encouraging trial of the device's capability and suggests that with refinements it could be utilised by disabled populations in physical therapy and to try to help their independence at home.

\section{REFERENCES}

[1] World Health Organization. (2013). The world health report [Online]. Available: http://www.who.int/whr/2013/report/en/

[2] M. D. Grossman and A. L. Stewart, ""You aren't going to get better by just sitting around": physical activity perceptions, motivations, and barriers in adults 75 years of age or older", Am J Geriatr. Cardiol., vol. 12, no. 1, pp. 33-37, 2003.

[3] J. H. Carr and R. B. Shepherd, A Motor Relearning Programme for Stroke. Oxford, GB: Butterworth-Heinemann, 1987.

[4] G. Kwakkel, B. J. Kollen, J. van der Grond and A. J. H. Prevo, "Probability of Regaining Dexterity in the Flaccid Upper Limb: Impact of Severity of Paresis and Time Since Onset in Acute Stroke", Stroke, Vol. 34, No. 9, pp, 2181-2186, 2003.

[5] G. A. Wolfe, "Burnout of therapists: inevitable or preventable?", Phys. Ther., vol. 61, no. 7, pp. 1046-1050, 1981.

[6] J. W. Berry and C. C. Hosford, "Burnout among physical therapist assistant program directors: a nationwide survey and analysis.", J. Allied Health, vol. 43, no. 2, pp. 72-78, 2014.

[7] U. Pustułka-Piwnik, Z. J. Ryn, Ł. Krzywoszański and J. Stożek, "Burnout syndrome in physical therapists - demographic and organizational factors.", Med. Pr., vol. 65, no. 4, pp. 453-462, 2014.

[8] E. G. Cruz, H. C. Waldinger and D. G. Kamper, "Kinetic and kinematic workspaces of the index finger following stroke", Brain, Vol. 128, No. 5,pp. 1112-1121, 2005

[9] M. Mulas, M. Folgheraiter and G. Gini, "An EMG-controlled Exoskeleton for Hand Rehabilitation", Rehabilitation Robotics, ICORR 2005, $9^{\text {th }}$ International Conference, pp. 371-374, 2005.

[10] E. G. Cruz and D. G. Kamper, "Use of a Novel Robotic Interface to Study Finger Motor Control", Annals of Biomedical Engineering, Vol. 38, No. 2, pp. 259-268, 2010.

[11] J. Allington, S. J. Spencer, J. Klein, M. Buell, D. J. Reinkensmeyer and J. Bobrow, "Supinator Extender (SUE): A Pneumatically Actuated Robot for Forearm/Wrist Rehabilitation after Stroke", Conf. Proc. IEEE Eng.Med. Biol. Soc., pp. 1579-1582, 2011.

[12] C. N. Schabowsky, S. B. Godfrey, R. J. Holley and P. S. Lum "Development and pilot testing of HEXORR: Hand EXOskeleton Rehabilitation Robot", Journal of NeuroEngineering and Rehabilitation, Vol. 7, No. 36, 2010.

[13] F. Zhang, L. Hua, Y. Fu, H. Chen and S. Wang, "Design and development of a hand exoskeleton for rehabilitation of hand injuries", Mechanism and Machine Theory, vol. 73, pp. 103-116, 2014.

[14] H. Kawasaki, S. Ito, Y. Ishigure, Y. Nishimoto, T. Aoki, T. Mouri, H. Sakaeda and M. Abe, "Development of a Hand Motion Assist Robot for Rehabilitation Therapy by Patient Self-Motion Control", Conf. Proc. IEEE Eng. Med. Biol. Soc., $10^{\text {th }}$ International Conferece, pp. 4282-4285, 2007.

[15] K. Kiguchi and Y. Hayashi, "An EMG-Based Control for an UpperLimb Power-Assist Exoskeleton Robot", IEEE Transactions on Systems, Man, and Cybernetics-Part B: Cybernetics, vol. 42, no. 4, pp. 10641071.

[16] H. Kim, L. M. Miller, Z. Li, J. R. Roldan and J. Rosen, "Admittance Control of an Upper Limb Exoskeleton - Reduction of Energy Exchange", Conf. Proc. IEEE Eng. Med. Biol. Soc., $34^{\text {th }}$ Annual International Conference, pp. 6467-6470, 2012.

[17] D. Jack, R. Boian, A. S. Merians, M. Tremaine, G. C. Burdea, S. V. Adamovich, M. Reece and H. Poinzer, "Virtual Reality-Enhanced Stroke rehabilitation”, IEEE Trans Neural Syst Rehabil Eng, Vol. 9, No. 3, pp. 308-318, 2001.

[18] A. S. Merians, D. Jack, R. Boian, M. Tremaine, G. C. Burdea, S. V. Adamovich, M. Reece and H. Poinzer, "Virtual Reality-Augmented 
Rehabilitation for Patients Following Stroke", Phys Ther, Vol. 82, No. 9, pp. 898-915, 2002.

[19] I. Kawabuchi, A Designing of Humanoid Robot Hands in Endoskeleton and Exoskeleton Styles, Humanoid Robots: New Developments. Vienna, Austria: I-Tech Education and Publishing, 2007.

[20] J. Li, R. Zheng, Y. Zhang and J. Yao, "iHandRehab: an Interactive Hand Exoskeleton for Active and Passive Rehabilitation", Rehabilitation Robotics, ICORR 2011, International Conference, pp. 1-6, 2011.

[21] V. Crocher, A. Sahbani, J. Robertson, A. Roby-Brami and G. Morel, "Constraining Upper limb Synergies of Hemiparetic Patients Using a Robotic Exoskeleton in the Perspective of Neuro-Rehabilitation", IEEE Trans Neural Syst Rehabil Eng, Vol. 20, No. 3, pp. 247-257, 2012.

[22] J.-U. Chu, I. Moon and M.-S Mun, "A Real-Time EMG Pattern Recognition System Based on Linear-Nonlinear Feature Projection for a Multifunction Myoelectric Hand", IEEE Transactions on Biomedical Engineering, Vol. 53, No. 11, pp. 2232-2239, 2006.

[23] Y. Su, M. H. Fisher, A. Wolczowski, G. D. Bell, D. J. Burn and R. X. Gao, "Towards and EMG-Controlled Prosthetic Hand Using a 3-D Electromagnetic Positioning System", IEEE Transactions on Instrumention and Measurement, Vol. 56, No. 1, pp. 178-186, 2007.

[24] D. Leonardis, M. Barsotti, C. Loconsole, M. Solazzi, M. Troncossi, C. Mazzotti, V. P. Castelli, C. Procopio, G. Lamola, C. Chisari, M. Bergamasco and A. Frisoli, "An EMG-controlled robotic hand exoskeleton for bilateral rehabilitation", IEEE Transaction on Haptics, Vol. 8, No. 2, pp. 140-151, 2015.

[25] Information on HAL-5 available at Cyberdyne website http://www.cyberdyne.jp
[26] R. A. R. C. Gopura and K. Kiguchi, "A Human Forearm and Wrist Motion Assist Exoskelton Robot with EMG-Based Fuzzy-Neuro Control”, Conf Proc IEEE Eng Med Biol Soc 2008, pp. 550-555, 2008.

[27] B. T. Iwamuro, E. G. Cruz, L. L. Connelly, H. C. Fischer and D. G. Kamper, "Effect of a Gravity-Compensating Orthosis on Reaching After Stroke: Evaluation of the Therapy Assistant WREX", Arch Phys Med Rehabil, Vol. 89, No. 11, pp. 2121-2128, 2008.

[28] S. W. Lee, K. A. Landers and H.-S. Park, "Development of a Biomimetic Hand Exotendon Device (BiomHED) for Restoration of Functional Hand Movement Post-Stroke", IEEE Transactions on Neural Systems and Rehabilitation Engineering, Vol. 22, No. 4, pp. 886-898, 2014.

[29] D. G. Kamper, T. G. Hornby and W. Z. Rymer, "Extrinsci flexor muscles generate concurrent flexion of all three finger joints", Journal of Biomechanics, vol. 35, pp. 1581-1589, 2002.

[30] R. C. Lyle, "A performance test for assessment of upper limb function in physical rehabilitation treatment and research", International Journal of Rehabilitation Research, vol. 4, no. 4, pp. 438-429, 1981.

[31] D. G. Kamper, B. D. Schmit and W. Z. Rymer, "Effect of muscle biomechanics on the Quantification of spasticity", Annals of Biomedical Engineering, vol. 29, pp. 1122-1134, 2001.

[32] D. G. Kamper and W. Z. Rymer, "A biomechanical simulation of the effect of the extrinsic flexor muscles on finger joint flexion”, IEEE Eng. Med. Biol. Soc., $23^{\text {rd }}$ Annual International Conference, Vol. 2, pp. 12561259, 2001.

[33] J. M. Ochoa, Y. Jia, D. Narasimhan, D. G. Kamper, "Developemtn of a portatble actuated orthotic glove to facilitate gross extension of the digits for therapeutic training after stroke", IEEE Eng. Med. Biol. Soc., $31^{\text {st }}$ Annual International Conference, pp. 6918-6921. 